\title{
LETTERS
}

\section{Statins in lymphangioleiomyomatosis: a word of caution}

\section{To the Editors:}

Lymphangioleiomyomatosis (LAM) is a rare progressive cystic lung disease affecting primarily females, characterised by the proliferation of neoplastic LAM cells with mutations in the tuberous sclerosis complex (TSC) 1 or TSC2 genes. There is no proven therapy for LAM, but results of studies with sirolimus, an inhibitor of the mammalian target of rapamycin (mTOR), have been promising [1]. Motivated by the finding [2] that in vitro, statins, which inhibit 3-hydroxy-3-methyl-glutarycoenzyme-A (HMG-CoA) reductase, inhibited the growth of $\mathrm{TSC2}^{-/-}$cells, we evaluated the effects of statins on the decline of lung function in 335 patients with LAM.

\section{METHODS}

We conducted a retrospective chart review on 504 patients with LAM referred to the National Institutes of Health since 1996, and enrolled in the National Heart, Lung, and Blood Institute (NHLBI) protocol 95-H-0186, approved by the NHLBI Institutional Review Board. All patients gave informed consent before enrollment. Patients were excluded if they were: 1) recipients of lung or kidney transplant; 2) receiving off-label sirolimus, or enrolled in the Multicenter International Lymphangioleiomyomatosis Efficacy of Sirolimus trial, or taking another mTOR inhibitor; 3) taking doxycycline [3]; 4) not followed long enough to calculate rates of decline in lung function; or 5) taking statins for an unknown length of time. Complete data for analysis were available for 335 patients. Univariate and multivariate analysis were conducted, comparing LAM patients receiving statins with the general LAM patient population.

In addition, we matched LAM patients receiving statins to LAM patient controls for variables known to affect decline of pulmonary function in LAM; i.e. age, number of years of follow-up, initial \% predicted diffusion capacity of the lung for carbon monoxide $(D \mathrm{~L}, \mathrm{CO})$ and forced expiatory volume in $1 \mathrm{~s}$ (FEV1) [4]. Analyses were performed using SAS v9.0 (SAS Institute Inc., Cary, NC, USA).

\section{FINDINGS}

Univariate analysis comparing 42 LAM patients taking statins with 293 LAM patients not on statins showed that LAM patients on statins were significantly older, had shorter followup time, lower initial $D \mathrm{~L}, \mathrm{CO} \%$, and a lower yearly rate of decline in FEV1 \% pred (table 1). The multivariate analysis, adjusting for age, showed that yearly rates of decline in FEV1\% pred were not significantly different in the two groups $(\mathrm{p}=0.718)$.

Of the 42 patients who were taking statins, we were able to match 37 patients to LAM patient controls. LAM patients

\begin{tabular}{|c|c|c|c|c|}
\hline \multirow[t]{2}{*}{ TABLE 1} & \multicolumn{4}{|c|}{$\begin{array}{l}\text { Baseline characteristics and lung function } \\
\text { decline of lymphangioleiomyomatosis patients } \\
\text { treated or not with statins }\end{array}$} \\
\hline & & Statin ${ }^{\#}$ & No statin & p-value ${ }^{+}$ \\
\hline \multicolumn{2}{|l|}{ Age yrs } & $53.1 \pm 1.09$ & $41.2 \pm 0.54$ & $<0.001$ \\
\hline \multicolumn{2}{|c|}{ Follow-up yrs } & $2.5 \pm 0.29$ & $4.3 \pm 0.17$ & $<0.001$ \\
\hline \multicolumn{2}{|c|}{ Initial FEV $1 \%$ pred } & $72.3 \pm 3.84$ & $79.1 \pm 1.48$ & 0.086 \\
\hline \multicolumn{2}{|c|}{ Initial DL,Co \% pred } & $65.7 \pm 3.40$ & $77.4 \pm 1.51$ & 0.003 \\
\hline \multicolumn{2}{|c|}{ Yearly change in FEV $1 \%$ pred } & $-0.4 \pm 1.06$ & $-2.4 \pm 0.33$ & $0.039^{\S}$ \\
\hline \multicolumn{2}{|c|}{ Yearly change in $D L, C O \%$ pred } & $-3.8 \pm 0.97$ & $-3.2 \pm 0.34$ & 0.549 \\
\hline
\end{tabular}

Data are presented as mean $\pm \mathrm{SE}$, unless otherwise stated. FEV1: forced expiratory volume in $1 \mathrm{~s}$; \% pred: \% predicted; $D \mathrm{~L}, \mathrm{CO}$ : diffusing capacity of the lung for carbon monoxide. ${ }^{*}: \mathrm{n}=42 ;{ }^{\bullet}: \mathrm{n}=293 ;^{+}$: unadjusted; ${ }^{\S}$ : statin was not a significant predictor of $F E V_{1} \%$ rate after adjusting for age $(p=0.718)$

receiving statins had lower minimum low density lipoprotein cholesterol levels than their matched controls, consistent with the statin inhibition of HMG-CoA reductase. Patients on statins had a statistically significant greater yearly decline in $\mathrm{DL}, \mathrm{CO} \%$ pred with a mean difference of $-3.7 \%$ (95\% confidence interval $-6.6--0.7 ; p=0.016$; table 2).

\section{DISCUSSION}

In addition to the beneficial effects of statins on lowering cholesterol levels and in the prevention of cardiovascular diseases, statin therapy has been advocated or is currently in clinical trials for a variety of lung diseases (e.g. pulmonary hypertension, sarcoidosis). However, a recent report details cases of statin-induced interstitial lung diseases [5]. Moreover, to date, no therapeutic success has been observed with statins in in vivo studies of TSC disease. Furthermore, statin treatment did not decrease cystadenomas size in $\mathrm{TSC}^{+/-}$mice [6]. There was no correlation between statin use and angiomyolipoma response to sirolimus in patients with TSC or sporadic LAM [1].

In the current retrospective study, we found that LAM patients on statins had a significantly greater yearly rate of decline of $D \mathrm{~L}, \mathrm{CO} \%$ pred than their matched controls. LAM patient controls had a relatively stable DL,CO over the follow-up period, which confirms earlier findings that rates of decline in lung function are lower in older post-menopausal females than in younger patients [4]. Two major limitations of the present study are; first, the small sample size, and secondly, as is inherent to all retrospective studies, the difficulty in establishing causality. 


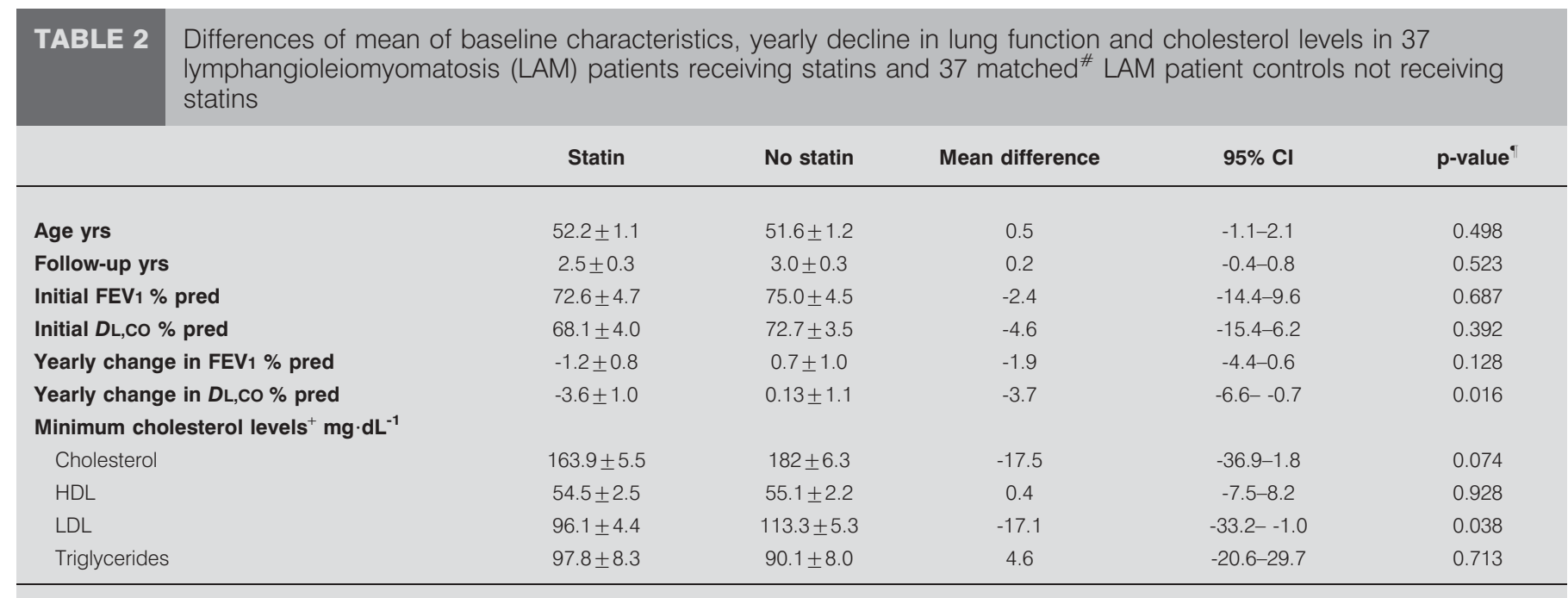

Data are presented as mean \pm SE, unless otherwise stated. Cl: confidence interval; FEV1: forced expiratory volume in $1 \mathrm{~s} ; \%$ pred: \% predicted; DL,CO: diffusing capacity of the lung for carbon monoxide; HDL: high-density lipoprotein; LDL: low-density lipoprotein. " ${ }^{\text {: }}$ matched for age, years of follow-up, initial DL,CO \% and FEV1; ": based on the paired t-test, some comparisons of lipids are based on fewer pairs $(n=32$ or 33$)$ due to missing values; ${ }^{+}$: lowest measured cholesterol over the follow-up period

The mechanism through which statins might increase the decline of lung function in LAM is unknown. However, elevated vascular endothelial growth factor (VEGF)-D levels were found in serum from LAM patients, and statins were reported to upregulate VEGF-D under normoxic conditions [7]. Higher levels of VEGF-D could enhance lymphangiogenesis in LAM nodules that contain lymphatic structures, and line lung cysts, thereby affecting DL,CO and not FEV1.

The decision to start patients with LAM on statins should be carefully weighed against the possibility of decline in lung function, and should probably not be done except as medically indicated or as part of a placebo-controlled trial.

\section{S. El-Chemaly*, A. Taveira-DaSilva*, M.P. Stylianou ${ }^{\#}$ and J. Moss*}

*Translational Medicine Branch, and \#Office of Biostatistics Research, National Heart, Lung, and Blood Institute, National Institutes of Health, Bethesda, MD, USA.

Correspondence: J. Moss, Translational Medicine Branch, National Institutes of Health, National Heart, Lung, and Blood Institute, Bldg. 10, Rm. 6D03, MSC 1590, Bethesda, MD 20892-1590, USA. E-mail: mossj@nhlbi.nih.gov

Support Statement: These studies were funded in part by the Intramural Research Program NIH/NHLBI (Bethesda, MD, USA).

Statement of Interest: None declared.

\section{ACKNOWLEDGEMENTS}

The current authors thank M. Vaughan (National Heart, Lung, and Blood Institute, National Institutes of Health, Bethesda, MD, USA) for helpful discussions and critical review of the manuscript.

\section{REFERENCES}

1 Bissler JJ, McCormack FX, Young LR, et al. Sirolimus for angiomyolipoma in tuberous sclerosis complex or lymphangioleiomyomatosis. N Engl J Med 2008; 358: 140-151.

2 Finlay GA, Malhowski AJ, Liu Y, et al. Selective inhibition of growth of tuberous sclerosis complex 2 null cells by atorvastatin is associated with impaired Rheb and Rho GTPase function and reduced mTOR/S6 kinase activity. Cancer Res 2007; 67: 9878-9886.

3 Moses MA, Harper J, Folkman J. Doxycycline treatment for lymphangioleiomyomatosis with urinary monitoring for MMPs. N Engl J Med 2006; 354: 2621-2622.

4 Taveira-DaSilva AM, Stylianou MP, Hedin CJ, et al. Decline in lung function in patients with lymphangioleiomyomatosis treated with or without progesterone. Chest 2004; 126: 1867-1874.

5 Fernandez AB, Karas RH, Alsheikh-Ali AA, et al. Statins and interstitial lung disease: a systematic review of the literature and of food and drug administration adverse event reports. Chest 2008; 134: 824-830.

6 Finlay GA, Malhowski AJ, Polizzi K, et al. Atorvastatin therapy does not affect the size or number of cystadenomas in $\mathrm{Tsc}^{+/-}$mice. Am J Respir Crit Care Med 2008; 177: A130.

7 Loboda A, Jazwa A, Jozkowicz A, et al. Angiogenic transcriptome of human microvascular endothelial cells: effect of hypoxia, modulation by atorvastatin. Vascul Pharmacol 2006; 44: 206-214. 